\title{
The Cognitive Use of Prior Knowledge in Design Cognition: The Role of Types and Precedents in Architectural Design
}

\author{
* Dr. Zeynep Çiğdem UYSAL ÜREY \\ Department of Architecture, Faculty of Architecture, Çankaya University, Ankara, Turkey \\ 'Email: zeynepuysal@cankaya.edu.tr
}

\begin{tabular}{|l|}
\hline A R T I C L E I N F O: \\
\hline Article history: \\
Received 15 October 2018 \\
Accepted 5 April 2019 \\
Available online 13 July 2019 \\
\hline Keywords: \\
Prior Knowledge; \\
Cognition; \\
Cultural Schemas; \\
Architectural Design; \\
Architectural Type. \\
\end{tabular}

This work is licensed under a Creative Commons Attribution - NonCommercial - NoDerivs 4.0. "CC @

\begin{abstract}
A B S T R A C T
This paper examines the cognitive use of prior knowledge in design and evaluates the role of types and precedents in architectural design and education from a cognitive perspective. Previous research on design cognition shows that the amount of prior knowledge possessed by the designer plays a fundamental role in the production and quality of the creative outcome. Prior knowledge is thought to be held by way of specific cognitive structures that are called cognitive schemas and, the role of our cognitive schemas (be it personal or cultural schemas) is portrayed as indispensable for the formation of our creative productions. Although significant efforts were made in the way of studying the use of prior knowledge in design, the correlation of types and cultural schemas has yet to be explored. This paper examines this correlation between cultural schemas, a markedly cognitive concept, and types, an architectural one, culminating in an investigation of the cognitive role of types and precedents within architectural design and education in the light of the cognitive literature. Building on that attempt, the study endeavors to conduct an interdisciplinary theoretical inquiry that respectively studies the role of prior knowledge in design cognition, the concept of cognitive-cultural schemas, the concept of type and its relationship with cultural schemas, and finally, the cognitive role of types and precedents in architectural design and education. In conclusion, this study proposes that, in terms of function, types are virtually identical to cultural schemas at the cognitive level, and types and precedents have a generative value for architectural design, by virtue of the fact that they exist as the initial cognitive schemas that are employed at the beginning of the design process.
\end{abstract}

JOURNAL OF CONTEMPORARY URBAN AFFAIRS (2019), 3(3), 39-50.

https://doi.org/10.25034/ijcua.2019.v3n3-4

www.ijcua.com Copyright (C) 2019 Journal of Contemporary Urban Affairs. All rights reserved.

\section{Introduction}

Empirical research on the cognitive properties of design has revealed a number of significant characteristics of the creative design process so far. One of such characteristics is about the nature of design problems. Design problems are portrayed as 'ill-defined' due to the fact that they often lack a clear definition, as their

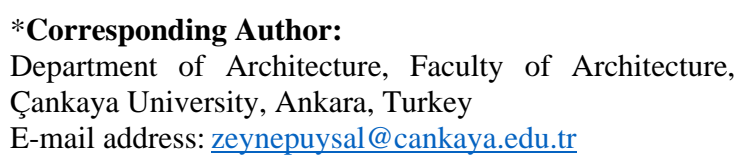


initial conditions, operations and goals are, at best, loosely defined and exposed to redefinition continuously. Design tasks in general, be it engineering problems or space planning tasks in architecture, are often reckoned as ill-defined due to these characteristics. The ill-defined design problems are shown to lack certain characteristics of well-defined problems, such as a wellspecified language for their representation, the initial knowledge of the generative manipulations to be applied on them, and a clear formulation of the goal state. These factors are found to be defined by their solvers, in other words, the designers (Cross, 2001, p. 3).

Research has revealed that unlike the ordinary problem solvers, the designers approach to illdefined design problems with a solutionfocused mindset, by means of constructing a problem 'frame' to demarcate the limits of the problem, propose a solution conjecture for it, and understand the problem through this solution (Cross, 2001, p. 3). Other than searching for the optimal solution, as would a problem solver handling a well-defined problem, designers are compelled to conceive a satisfactory solution for solving the ill-defined design problem. Doing so, the designers look for a match between the problem and the solution, and perceive and decipher the problem through these solution perspectives. In order to get a working match in this problem-solution pair, the designers are found to employ primarily their prior knowledge. As Nigel Cross (2006) explains it, creative design does not always occur in this sense as the proposition of an unanticipated and unusual proposal, but often as the making of a suitable proposal, which contains novel features for a new design product. It is believed on this basis that creative design takes place via a 'creative leap' from the design constraints to the solution proposal, which is supported by the prior knowledge of designers (Cross, 2006, p. 3, 44).

\section{Cognitive Use of Prior Knowledge in Architectural Design}

As indicated above, the solution-oriented mindset underlying design, builds essentially on the prior knowledge of designers. Described as "a particular structured formulation of underlying types such as concepts, prototypes and precedents", prior knowledge is widely recognized as an intrinsic element of any creative design process
(Oxman, 1999, pp. 17-28). As McDermott (1982) put it, given the understanding that design is an ill-structured activity, and that the set of constraints applicable to specific design problems is often substantial, one can hope to surmount these problems only when significant volumes of domain specific knowledge can be combined and fused together at every stage of the problem solving process (p. 36). In this context, ill-defined design problems necessitate nothing but a large base of appropriate prior knowledge for the formation of their solutions.

As Jansson, Condoor \& Brock (1992) study shows, at the early phase of design, namely the representation of the problem, the prior knowledge of designers, in the form of prototypes, types, or precedents, serves as a cognitive point of reference to start the design process. At the start of the design process, the designers are considered to analyze existing systems looking for analogies. They then proceed to bring up a first solution concept that acts as the starting point from which to tackle the design problem they face with. In this process, the designer focuses on the smaller parts of the wider problem, by means of sub problems, using a retrieval system that continuously recalls prior knowledge from his/her long-term memory. Since ill-defined design problems require substantial amounts of relevant prior knowledge, the retrieval system employed on them operates as a device to recognize the solution alternatives. As the design problems are downsized to a series of sub problems as such, these smaller parts can be handled better as well-defined problems (Fig. 1) (Simon, 1973, pp. 181-201).

Likewise, Bonnardel and Marmeche (2005) suggest that the designer's past experiences, which are stored in terms of his/her prior knowledge, are often the sources of inspiration in the formation of new ideas (pp. 422-435). In this sense "designers accommodate the known to the new" and thus develop the new ideas through integration with "what they already know" (Oxman, 1990, p. 23). To Oxman (1990b), design occurs in this sense as "a dynamic process of adaptation and transformation of the knowledge of prior experiences in order to accommodate them to the contingencies of the present" (1990b, pp. 17-28).

In this context creativity in design occurs as "the sudden interlocking of two previously unrelated, skills or matrices of thought" (Koestler, 1964, p. 121), and emerges as a 
cognitive process entailing the "activation and recombination of previous knowledge elements in a new way in order to generate new properties based on the previous ones" (Bonnardel \& Marmache, 2005, pp. 422-435). Thus, the studies on design creativity show that people depend mostly on past experiences, types and precedents, even when they are instructed to be as original and imaginative as possible. In this perspective, the new ideas that are developed are deemed creative and original to the extent that they move away from their initial sources of inspiration (Bonnardel \& Marmache, 2005, p. 422-435). To quote from Suwa, Purcell \& Gero (1998), design can be seen on this basis as "a kind of apprenticeship in which skills and expertise are acquired after learning basic techniques, assimilating domain specific and general knowledge, and inspecting past good examples" (p. 455). Today, it is widely accepted that the design ability grows in parallel with the extent of the acquired domain knowledge and the problem solution strategies that are operated on that knowledge. The obvious implication of this information is that, if designers or students of design are provided with ever growing databases consisting inter- or intra-disciplinary sources, their success in producing creative designs would only increase (Bonnardel \& Marmache, 2005, pp. 422-435). They would be expected to produce better outcomes with the provision of mental cues given in the form of previous designs that show them the use of design elements and how they can be combined in individual settings (Malhotraa, Thomas, Carroll \& Millera, 1980, pp. 119-140).

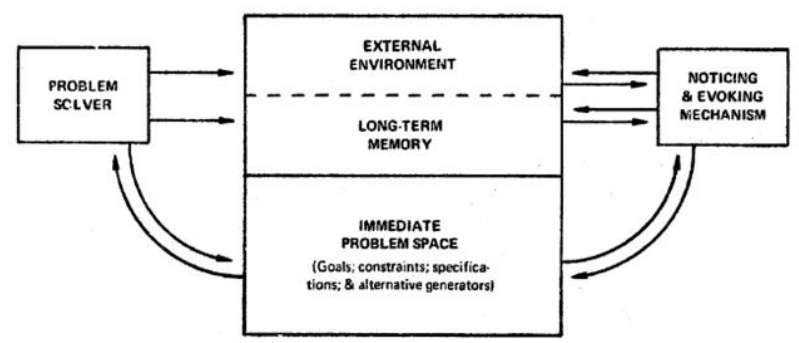

Figure 1. A model for ill-structured problems (Simon, 1973).

\subsection{Types of Prior Knowledge}

According to the literature, there are essentially two types of prior knowledge that are employed in design: the declarative (or the domain-specific) knowledge, and the procedural knowledge (Goel, 2001). Declarative knowledge refers to the general knowledge about the 'things' that we learn within our lifetime and it essentially consists of the 'facts' that we know. Procedural knowledge, on the other hand, involves the procedures used for the processing of the declarative (or the domain-specific) knowledge (Goel, 2001, pp. 221-241). In the context of performing a given task, the declarative knowledge is often believed to go through a transformation into a procedural form (Chan, 1990, p. 62). As Purcell and Gero (1991) put it, these types of prior knowledge are acquired by means of either exposure to incidental experiences, which characterize everyday life, or as a result of intentional learning, in which domain-specific experiences and the strategies employed for their transformation are instilled in the subjects by means of education (p. 82).

Prior knowledge is also categorized as personal or cultural in terms of its source. If prior knowledge emerges exclusively on the basis of the specific, personal experiences of the individual who possesses it, it is considered personal. On the other hand, if it is formed by a multitude of phenomena shared by the wider society, or at least a community, it is deemed as cultural (Shore, 1996, pp. 56-65). Architectural types for instance are the cultural forms of this prior knowledge.

\subsection{Prior Knowledge and Cognitive Schemas in Design}

The cognitive literature states that prior knowledge is held by our minds by way of those specific cognitive structures that are called cognitive schemas. Described as the conceptual structures that stand for our knowledge of situations, events, objects and actions (Wertsch, 1985, p. 154), they are defined as the mental frameworks that we make use of in the organization of our knowledge. They are the conceptual structures that organize and direct our reception, storage, retrieval and production of information (D'Andrade, 1992, p. 28). Operating in long-term memory as organized structures of knowledge, they guide perception, enable comprehension and direct thinking. By schema theory, what the cognitive literature demonstrates us is the importance of our prior knowledge in the understanding of the forthcoming information and in the formation of new knowledge (Bruning, Schraw, Norby \& Ronning, 2004, p. 6, 22, 23).

As the bearers of prior knowledge, cognitive schemas are also categorized as personal or cultural in terms of their source. Personal 
cognitive schemas are formed through personal experiences and are specific to individuals. Cultural schemas, on the other hand, are attained through the individual's relationship with the cultural context and are specifically defined as the "patterns of basic schemas that make up the meaning system of a cultural group" (Nisbett \& Norenzayan, 2002, p. 5,6$)$. They are shared by the members of the society and they regulate their daily experiences and the interpretation of these experiences. As Holland \& Quinn (1987) explain, they are the "presupposed, taken-forgranted models of the world that are widely shared by the members of a society and that play an enormous role in their understanding of that world and their behavior in it" (p. 4). As situated cognition theory states, people carry their load of cultural information and operate them through their cognition by way of their cultural schemas, which exist as a subset within their bigger collection of cognitive schemas (Johnson, 1987, p. 19; Oyserman, Sorensen, Reber \& Chen, 2009, p. 219).

Cognitive schemas are believed to encompass both the knowledge itself (declarative knowledge) and the information on the actual operationalization of that knowledge (procedural knowledge) (Chan, 1990, p. 62). In this sense, as means to carry generic information, cognitive schemas provide the most efficient and most widely used type of information in the field of design. They contain information about objects, their constituents, and the relations between those constituents (Purcell \& Gero, 1991, p. 83). Describing schemas in design as "the formal constructs for capturing, acquiring and representing types of knowledge structures in design", Oxman (2004) argues that schemas constitute the essence of conceptual design knowledge, which, in turn, is structured around a number of conventions including typologies, rules, or precedents (p. 70). Looking for a relevant schema to organize our prior knowledge around in line with the cause at hand is considered as an essential part of the creative design process (Oxman, 1990). In this context, design creativity is seen as "the ability to innovatively re-represent the schema or the particular structural content of the externalized representation" (Oxman, 1996, p. 333).

Therefore, prior knowledge in architecture and design, attained through experience or education, is held by way of the cognitive schemas of the designer, in forms such as types, prototypes or precedents (Lawson, 2004 , p. 443). These schemas are used in the design process for recognizing the design situation, in the same way as the chess masters recognize the situation in a chess board by means of their experience. The recognition process accelerates and facilitates the design response, as it enables a quicker analysis compared to an in-depth analysis, and allows the designer to develop a solution by employing a standard gambit (Lawson, 2004, p. 448). The schemas are used as the 'cognitive reference points', which provide the first solution concepts that initiate the design (Goldschmidt, 1998; Jansson, Condoor \& Brock, 1992). By providing the first solution concepts and supplying the gambits that are previously developed by the designer to solve similar problems, schemas hook up the design problem to its solution. The 'gambits' that are utilized on the existing schemas, either to transform them or adapt them to the cause, are described as the 'repertoire of tricks' or the applicable design strategies, which are used by the designer/architect to solve recognized problems. They are essentially the techniques used for the creation and transformation of forms, and designers often exhibit variance in terms of how they employ them (Lawson, 2004, p. 448).

Jansson et. al. (1992) defines three cognitive processes that are active in this process of using schemas (or prior knowledge) in architectural design: identification, where designers use types or prototypes for categorizing, understanding and representing design problems; synthesis, where they adapt or transform types or prototypes so as to fulfill the requirements associated with the problem; and evaluation, where they assess themselves with reference to those types or prototypes. These processes are believed to work in a consecutive and repetitive manner towards the realization of a complete architectural design (Jansson, Condoor \& Brock, 1992).

According to the literature, one needs a certain level of maturity to practice design well, as expertise in design is essentially a culmination of design knowledge and experience in the form of schemas. Lawson (2004) indicates that, the educational period of the designer helps him/her to develop a knowledge of design solutions, in the form of 'the pool of precedents' or the 'domain specific knowledge' (p. 456). The designers 
who are considered experts are characterized by a vast pool of precedents and prior knowledge, which are stored as solution schemas to be employed at different design projects (Lawson, 2004, p. 456). For expert designers, the schemas of precedents or types do much more than just carrying the visual information and geometry. They also convey all the concepts related to that schema, including but not limited to the materials, functions, organization principles, and significant instances of that schema (Lawson, 2004, p. 443). The schemas of expert designers are observed to be greater in number, in detail and in the extent of information that they hold (Purcell \& Gero, 1991, p. 83). Lawson (2004) defines five stages, which the designer has to undergo in his/her journey to gain expertise in design:

1. Formation of a developing pool of precedents

2. Attainment of design schemas

3. Development of certain guiding principles (e.g. sustainable design)

4. Development of the skill of recognizing the design situation without the need of an in-depth analysis

5. Formation of design gambits or a 'repertoire of tricks' that are fused within the schemas used for recognizing the design situations (Lawson, 2004, p. 456-457)

In this context, it is evident that the designers ought to examine a considerable amount of types and precedents in order to grow their load of schemas, which would enable them to "recognize underlying structures in design situations" and allow them to "employ and adapt gambits" that they acquired earlier (Lawson, 2004).

\section{Two Types of Domain Specific Prior Knowledge in Architectural Design: Types and Precedents}

According to Oxman (1992), the prior knowledge utilized in architectural design can be categorized into two groups: the types and the precedents. In this categorization, types and precedents are different from each other in terms of the form of reasoning that they demand from the designer: the former requires 'refinement' while the latter demands 'adaptation'. Employed as two distinct cognitive approaches to design, they are related with typological (model based) generic design and precedent-based (casebased) adaptive design, respectively (Oxman,
1992, p. 117). The section below will provide a glance at these two types of domain specific prior knowledge, so as to evaluate their role in architectural design.

\subsection{Types}

The formation of types, or the process of typification, is an outcome of the process of generalization or categorization. Oxman (1990b) describes typification as "the abstraction and classification of salient aspects of precedents in terms of both situations and solutions" (p. 17-28). One of the most evident applications of this process is formal typification, where classes of formal types are produced as based on certain known precedents. Typification also occurs in the perception of the design problem, where the designer tries to match the problem with a similar solution type that he/she previously encountered with. Regarding this process, Oxman (1990b) goes as far as claiming that "design knowledge is the knowledge of typification through abstraction" (p. 17-28):

"We assume that all design experience undergoes processes of typification in order to create indices for the storage, and ultimately for the retrieval, of design episodes; and that the way in which this occurs is a function of the form of classification and existing structure of the designer's memory." (Oxman, 1990, p.

24)

In this sense, typology operates as a form of indexing and categorization in design. Oxman (2001, 2004) describes typological knowledge as "a set of generic representations which are associated with specific problem types" (Oxman, 2001, p. 278) and defines types as the "conventionalized knowledge structures" (Oxman, 2004, p. 70) that occur as an important form of knowledge representation for the studies of design cognition. Types are considered as the characteristic forms of domain specific architectural knowledge that are attained by the designers through education and personal experience (Oxman, 1996, p. 332). They contain a mass of prior knowledge allowing the designer "to extract generic schema from specific images" (Oxman, 2001, p. 280), and consist of both the 'generic representational schema', as well as the knowledge of the strategies to employ when using this schema. In types, Oxman 
(1990a) sees the formalizations of a high level of design knowledge encoded in generic forms, and does not refrain from calling them the general solution schemas, which act as the sources of generic knowledge to be manipulated in the process of design (Oxman, 1990, pp. 2-8).

In the light of the research on design cognition, architectural types could be seen as a part of our store of cultural schemas. Being the cultural attributes that are shared by a society, types behave like cultural schemas on the cognitive level, assisting both the interpretation of incoming architectural information and also the production of new designs (Oxman, 1990 pp. 2-8). Architectural theory refers to type both as an abstract conceptual form, as well as a cognitive facility, which provides the background for the systemic action of design that nestles essentially on categorization (Habraken, 1985, p. 40). As Moneo defines it, type could be seen in this sense as:

"...the concept which
describes a group of objects
characterized by the same
formal structure. It is neither a
definite spatial diagram not
the average of a serial list. It is
fundamentally based on the
possibility of grouping objects
by certain inherent structural
similarities. It might even be
said that type means thinking
in groups." (Moneo, 1978, p.
23)

The elimination process that sort out only the common elements that belong to the group in question makes type "a schema and a collective product that is shared both by the architects and the community they serve to" (Petruccioli, 1998, p. 11). As Quatremere de Quincy's definition also shows, type is "neither a concrete image of something that can be copied directly, nor it is a definite form, but it is a schema or the outline of a form, which acts as the abstract structure used for spatial articulation" (cited in Argan, 1996, p. 240, 244). In this line of thought, architectural type can be understood as a 'schema of spatial articulation', which is shaped, if not formulated, as an answer to the ideological and practical needs of a society (Argan, 1996, p. 246). It functions as a non-linguistic cultural schema, associated with a specific society, and provides a visual image or a virtual model of a culture (Shore, 1996, pp. 56-65). It operates both in the interpretation of architectural products, as the preliminary schema of reference, and also in their production, as the purveyor of thought towards creative manipulations for new designs.

According to Oxman (1990b), new designs could be built upon prior design knowledge and experiences, due to the fact that those experiences are abstracted, encoded and categorized in the form of types in long term memory. With their abstract and generic formation, types are capable of various new design solutions and in this process, analogical thinking stands out among other cognitive processes with the lead part that it plays (Oxman, 1990, p. 17-28).

Cognitive schemas are used both for the representation of the typological knowledge that they carry in the mind, and for its processing for the purpose of coming up with a generic design (Oxman, 2001, p. 278). In Oxman's jargon, the reasoning or processing style employed in the context of utilizing types in design is called as 'refinement'. 'Typological refinement' basically refers to the distinctive thinking style employed in the formal processing of typological knowledge in design. The form of creativity emerging in this process on the other hand, is called as 'typological emergence'. Oxman duly provides an example of this notion with an illustration, showcasing type use in chair design, as part of the extended process of typological emergence (Fig. 2) (Oxman, 2001, p. 278).

In refinement, the original state of a generalized (generic) schema, which is the architectural 'type', is successively transformed into a specific design through a top-down process. This underlying schema is called 'generic' due to the fact that it consists of only the most significant properties of the class of designs it belongs to and the type of design that refines this generic schema of type is called as the generic or the typological design (Oxman \& Oxman, 1992, p. 119). Oxman accordingly developed a model that expresses the cognitive processing of generic or typological design, entailing the relationship between the design issue (the specification of the problem), design concept (the type of the solution) and design form (the end result). As shown by this model, the design process starts with deciding on a solution class (type), followed by developing the first form of generic representation of this class (level 1, 
which is basically the first modification of the type), and finally culminating in the realization of the solution form (Fig. 3-5) (Oxman, 1994, pp. 141-146) (Oxman, 2001, p. 284). According to Oxman (1990b), in design, types could either be refined, by applying consecutive alterations over them to create new designs (appropriation), or could be adapted, by making greater formal or functional modifications over them to reach to new designs (analogy). Yet, a third alternative is also proposed to account for the cases where the existing types are not suitable for the situation and when completely new types are generated by, once again, using existing knowledge (innovation) (Oxman, 1990, pp. 1728). In this process, the design constraints function as the source of transformations, modifications or the generation.

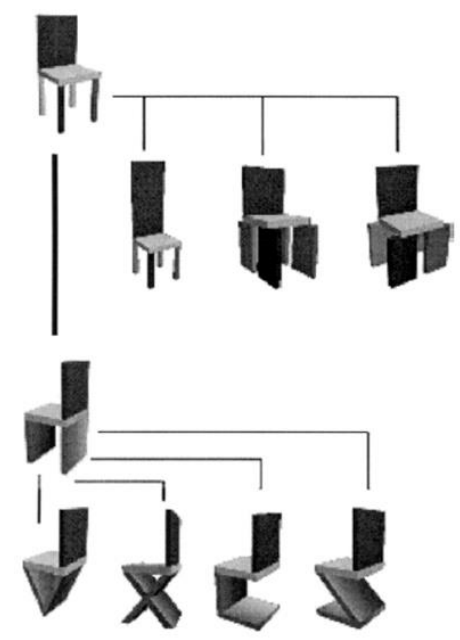

Figure 2. The process of the creative transformation of type through typological emergence. (Oxman, 2001, p. 279)

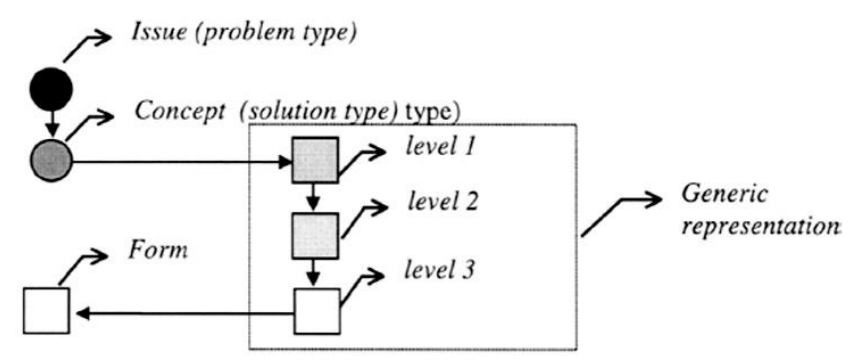

Figure 3. The steps of design thinking in generic or typological design (Oxman, 2001, p. 285)

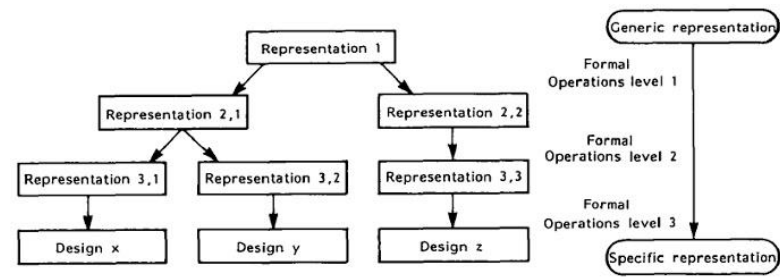

Figure 4. The refinement of a generic schema (type) in design (typological design). (Oxman, 1992, p. 122)
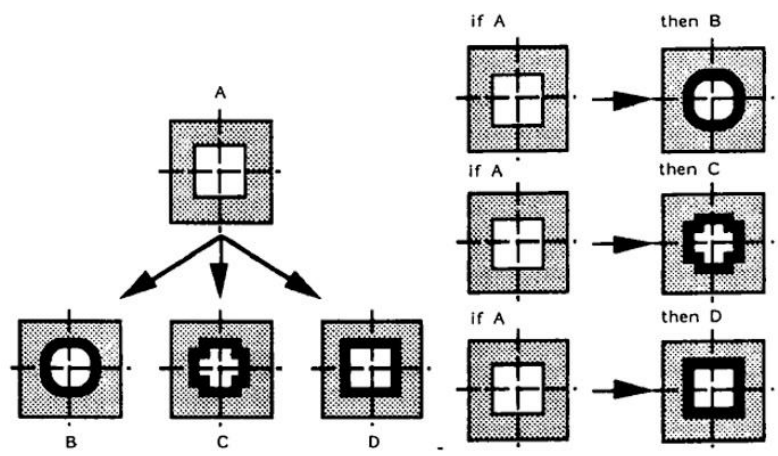

Figure 5. An example to the refinement process. (Oxman, 1992, p. 123)

\subsection{Precedents}

The other significant type of domain specific knowledge available for the use of designers in new design contexts is the knowledge of design precedents (Oxman, 1996, p. 332). Within the context of creative design, designers evidently use cases or the knowledge of applicable prior designs to solve the recent problems they face with (Akin, 2002, p. 2). In their quest for new design ideas, designers look through numerous precedents to form applicable connections with the problem and this activity is often credited with enabling the appearance of new and previously unforeseen ideas for the designer (Oxman, 1994, p. 141, 142).

Precedent is described as "the design case knowledge, which includes the particular conceptual contribution to design, which makes a case memorable as a precedent" (Oxman, 1994, pp. 141-142). To put it another way, precedents are the "specific designs or buildings, which are exemplary in some sense so that what architects and students glean from these examples, can support their own designs" (Akin, 2002, p. 3). In simpler terms, they are the earlier solutions to particular design problems. They are essentially different from types due to the fact that they are the specified design representations, instead of being the abstract schemas (Fig. 6) (Oxman, 2001, p. 284). 


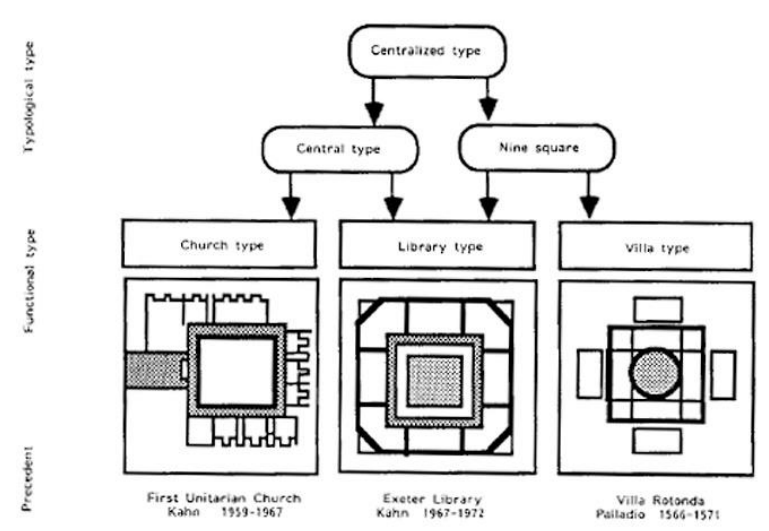

Figure 6. Precedents, functional types, high level typological concepts. (Oxman, 1990b, p. 23)

People are believed to benefit from precedents by mining the information they contain through analysis and abstraction. According to Akin, the conceptual abstractions acquired from precedents enable the designers to crossover the chasm between the conceptual and the physical design environment (Akin, 2002). This appears as the main reason behind the fact that solving problems in design requires not only the "problem solving skills and strategies" but also a large "body of knowledge", which allow the application of these skills to specific problems (Akin, 2002). Precedents are found to be helpful in this sense due to the fact that they contain and display both the processes and the products of design within themselves. In this framework, it is not surprising to see that the studies on design education portray the knowledge of design precedents, and the concepts that are attained through them, as one of the most important sources of knowledge in design education (Oxman, 2004, p. 71).

Precedent knowledge is employed in design by means of 'case-based reasoning'. This reasoning type essentially refers to the use and application of past experiences and examples for the understanding, analysis and solution of current problems. It is the process of choosing relevant ideas from prior problems for application to the current ones. According to Kolodner (1992), in case-based reasoning, the problem solver recalls a previous case that resembles the current one, or that is similar to it in certain aspects, and utilizes the solution to that past case to solve the case at hand (pp. 3-4). In this process, the reasoner may go with adapting the old solutions to meet the demands of the new problems in order to solve them and this becomes the common practice of "problem solving type of case based reasoning' as embraced by the designers. Or the reasoner may engage with the previous cases to explain, interpret and critique a current case, thus taking the route of 'interpretive type of case-based reasoning', as lawyers often do (Kolodner, 1992, pp. 3-4).

'Problem solving type of case based reasoning' is employed very frequently in design. The process of choosing relevant ideas from prior designs, which can be applied effectively to the design problems at hand, is called as precedent-based (or case-based) design. What the precedents used in casebased design do is to provide 'a vocabulary of design ideas' and the process that is used to transform these ideas to make them applicable to current cases is called as 'adaptation' (Oxman, 2001, p. 284). According to Oxman (1996), adaptation is essentially the process of "fitting the old solution to a new one, or evolving a new design by modifying an existing solution representation" (p. 334). It involves the re-use of the prior representational content of an existing design solution after a thought out modification (Oxman, 1996, p. 334). The precedents, which are deemed to be stored in the form of cognitive schemas in the mind, are processed through adaptation for the formation of new design solutions (Fig. 9) (Oxman, 2001, pp. 269-295).

As Kolodner (1992) suggests, case-based reasoning in design offers a complete solution for the design problem at hand and any pieces of the previous solution which do not fit the current problem can be adapted to it later. Although the amount of adaptation and the effort required to tailor the solution to the current problem might be substantial, and at times overwhelming, this method is almost always preferable to starting from scratch, which is often an intimidating task. It facilitates the design by enabling the designer to avoid numerous constraints and saving her from the need to compartmentalize the problem into multiple parts just to avoid the inherent difficulty of handling a larger problem (Kolodner, 1992, pp. 5-9). According to Kolodner (1992), case-based reasoning occurs in four steps (Fig. 8):

1. The accumulation of precedents or experiences

2. Remembering prior experiences that are similar to the current case at hand. and interpreting the new case in the light of these prior experiences through comparison 
3. Adaptation of the prior experiences to "fix up an old solution to meet the demands of the new situation"

4. Evaluation and repair of the outcomes

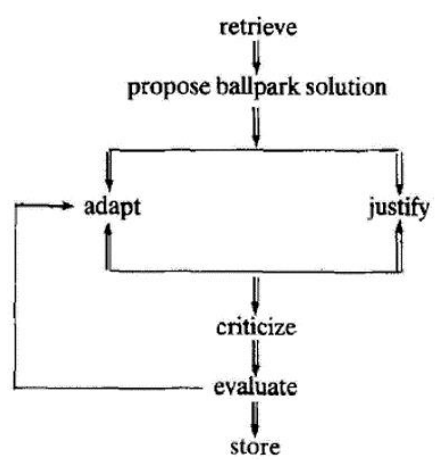

Figure 7. Case-based reasoning cycle (Kolodner, 1992, p. 22)

\section{Prior Knowledge in Design Education}

The studies on prior knowledge evidently affected the design education as well. In 1969, Laxton (1969) developed a model for design education, which began by the accumulation of the reservoir of knowledge and experiences, followed by the development of the ability to develop ideas, and culminated by the acquisition of the skill of critically evaluating the developed ideas so as to interpret and transform them to meet the requirements of new contexts. He stated that design education at schools should entail, first and foremost, the domain specific knowledge of precedents, as the students cannot be realistically expected to be creative without accumulating a 'reservoir of knowledge' first, which would serve them as a pedestal on which to rise. According to Laxton (1969), the ability to develop new ideas is essentially contingent on this reservoir of knowledge to be filled well. In Lawson's (2004) view, this model of design education was based principally on the development of prior knowledge and experience, rather than the generation of new ideas by way of a tabula rasa attitude, which was the dominant perspective towards the design education in the 20th century, valuing originality above all (p. 454).

More recently, Akin called the method of education with a marked emphasis on the teaching of the precedents as "case-based instruction" (Akin, 2002). Based mostly on the analysis of precedents, this approach to education is expected to demonstrate the students the principles and strategies of architectural design by means of cases. The students are supposed to learn the design heuristics with the help of the precedents that they have examined. Even though this approach is criticized with the claim that it restricts the creative capacity of students, the literature offers no concrete evidence to prove the validity of this claim (Akin, 2002).

By far, the studio based education is currently the most extensively employed method in schools of design. This method essentially tries to simulate the context of a professional design office and to replicate the actual phases of the design process, for instance the esquisse phase or the jury system, that are experienced in a design office (Oxman, 1999, pp. 105-106). This education offers an experience-based learning, where the student engages in design activity under the supervision of the instructor (Oxman, 2004, p. 110). The students are not offered a didactic education focusing on abstract principles to be applied to problems, but an experiential one that depends on the hands on problem solving experience gained by dealing with specific design problems at hand (Akin, 2002, p. 2).

According to Oxman, this system should be enhanced methodologically to enable the students to attain the domain knowledge of design, by means of cognitive schemas such as types or precedents, and the strategies of design thinking such as analogy, refinement or adaptation, which can then be used to manipulate these cognitive schemas to handle the specifics of the current problem (Oxman, 2004, p. 110):

"It is our hypothesis that learning in design is the acquisition of the cognitive ability to manipulate the representations of design knowledge, to acquire basic schema in design thinking, to understand knowledge structures and to be able to manipulate characteristic strategies of design thinking such as generic and typological design, adaptive design, analogical thinking and creative exploration. That is, the cognitive attributes of design cognition and learning can become the content of design education." (Oxman, 2004, p. 110) Under the guidance of several cognitive studies on design, Oxman identifies the necessary constituents that a design education should support the student with (Oxman, 2001, p. 280): 
1. Cognitive structures: The attainment of the cognitive ability to represent design knowledge via basic schemas of design thinking, such as types and precedents, and,

2. Cognitive strategies: The attainment of strategies, such as refinement or adaptation, to transform these basic schemas in order to produce original solutions.

It becomes evident by this point that an effective design education should be based on an amalgamation of two distinct components: the domain knowledge (or vocabulary) of design that the students should learn by examining types and precedents; and the strategies to be employed in the design process that the students should develop by hands on design exercises (Oxman, 2004, p. 65). An educational approach capable of providing these two components is believed to bestow the student with the ability of the 'designerly way of thinking' (Cross, 2006).

\section{Conclusion}

The studies discussed so far demonstrate that the designers' prior knowledge has a crucial impact in the initiation of the design process and in the production of new designs. Cognitive schemas that store this prior knowledge within our minds appear as the main actors in this process and they play a formative part both in the interpretation of incoming design information and also in the production of new ones. Through this schematic structure of our minds that is essentially based on learned information, we are intrinsically bound to our prior knowledge for the scope of our design ability. The comparative study of the literature on design cognition and architectural theory further demonstrates us that there is an effective correlation between cultural schemas and architectural types. It is observed that architectural types function identically as cultural schemas at the cognitive level, and types and precedents have a generative value for architectural design, by virtue of the fact that they exist as the initial cognitive schemas working at the start of the design activity.

On these terms, studies on the cognitive use of prior knowledge in design provide a framework for us to conceive creativity differently by viewing culture from the lens of cultural-cognitive schemas, that of types and precedents. Suggesting that creativity in design essentially stems from familiar forms and methods of production, studies reject the idea that creativity is the "creation of something out of nothing'. On the contrary, they propose that creative production in design is about the production of something new through the refinement, adaptation, recombination and transformation of existing knowledge. Within this proposed perspective, the role of the familiar, the known and the existing, as embodied by types and precedents, occurs as important as the novel, the unknown, and the prospective, for the realization of creative design.

\section{Acknowledgement}

This work makes partial use of some of the theoretical discussions about the use of prior knowledge in architectural design in my $\mathrm{PhD}$ Dissertation (Uysal, Zeynep Çiğdem, 2012). Architectural Type as a Cultural Schema and Its Cognitive Use in Architectural Design: An Analysis of the Aga Khan Award Winning Dwellings in Turkey (1970-2008), Unpublished $\mathrm{PhD}$ Dissertation, North Carolina State University, Raleigh. Supervisor: Prof. Kristen Schaffer) and on this account I would like to express my sincere gratitude once again to my advisor Prof. Kristen Schaffer and my committee members Prof. Paul Tesar, Prof. Meredith Davis, and Prof. Ronald Endicott.

\section{Conflict of interests}

The Author declares that there is no conflict of interests.

\section{References}

Akin, O. (2002). Case-based instruction strategies in architecture. Design Studies. 23. 407-431. https://doi.org/10.1016/S0142694X(01)00046-1

Argan, G. C. (1996). On the typology of architecture. In K. Nesbitt (Ed.), Theorizing a New Agenda for Architecture: An Anthology of Architectural Theory 19651995. New York: Princeton Architectural Press. https://www.amazon.com/TheorizingNew-Agenda-ArchitectureArchitectural/dp/156898054X

Bonnardel, N., \& Marmeche, E. (2005). Towards supporting evocation processes in creative design: A cognitive approach. International Journal of Human Computer Studies, 63, 422-435. http://dx.doi.org/10.1016/j.ijhcs.2005.04.006 
Bruning, R. H., Schraw, G., Norby, M. \& Ronning, R. (Eds.). (2004). Cognitive psychology and instruction. Columbus, Ohio: Pearson Merrill Prentice Hall.

Chan, C. S. (1990). Cognitive processes in architectural design problem solving. Design Studies, $11(2)$, 60-80. https://doi.org/10.1016/0142-694X(90)90021$\underline{4}$

Cross, N. (2001). Design cognition: Results from protocol and other empirical studies of design activity. In C. Eastman, W. Newstetter \& M. McCracken (Eds.), Design knowing and learning: Cognition in design education. Oxford: Elsevier Books. https://www.elsevier.com/books/designknowing-and-learning-cognition-in-designeducation/eastman/978-0-08-043868-9

Cross, N. (2006). Designerly ways of knowing. London: Springer-Verlag. https://www.springer.com/la/book/9781846 283000

D'Andrade, R. (1992). Schemas and motivation. In R. D'andrade \& C. Strauss (Eds.), Human motives and cultural models. Cambridge: Cambridge University Press. DOI:

https://doi.org/10.1017/CBO9781139166515. $\underline{003}$

Goel, V. (2001). Dissociation of design knowledge. In C. Eastman, W. Newstetter \& M. McCracken (Eds.), Design knowing and learning: Cognition in design education. Oxford: Elsevier Books. https://www.elsevier.com/books/designknowing-and-learning-cognition-in-designeducation/eastman/978-0-08-043868-9

Goldschmidt, G. (1998). Creative architectural design: reference versus precedence. Journal of Architectural and Planning Research, 15(3), 258-270. https://www.jstor.org/stable/43030466

Habraken, N. J. (1985). The appearance of the form: four essays on the position designing takes place between people and things. Cambridge, MA: Awater Press. https://www.amazon.com/AppearanceForm-Position-Designing-Betweenebook/dp/BOOIOVOUQC

Holland, D. \& Quinn, N. (1987). Culture and cognition. In D. Holland \& N. Quinn (Ed.s), Cultural models of language and thought. Cambridge: Cambridge University Press. https://www.amazon.com/CulturalLanguage-Thought-DorothyHolland/dp/0521311683
Jansson, D. G., Condoor, S. S. \& Brock, H. R. (1992). Cognition in design: viewing the hidden side of the design process. Environment and planning b: planning and design, 19, 257 - 271. https://doi.org/10.1068/b200257

Johnson, M. (1987). The body in the mind: The bodily basis of meaning, imagination and reason. Chicago: University of Chicago Press.

https://www.press.uchicago.edu/ucp/book s/book/chicago/B/bo3613865.html

Koestler, A. (1964). The act of creation. London: Penguin Books. https://www.amazon.co.uk/Act-CreationArthur-Koestler/dp/1939438985

Kolodner, J. L. (1992). An introduction to casebased reasoning. Artificial intelligence review 6, 3-34. http://dx.doi.org/10.1007/BF00155578

Lawson, B. (2004). Schemata, gambits and precedent: some factors in design expertise. Design studies, 25(5), 443-457. https://doi.org/10.1016/j.destud.2004.05.001

Laxton, M. (1969). Design education in practice. Attitudes in design education. London: Lund Humphries.

Malhotra, A., Thomas, J. C., Carroll, J. M. \& Millera, L. A. (1980). Cognitive processes in design. International journal of manmachine studies, 12(2), 119-140. https://doi.org/10.1016/500207373(80)80013-7

Mcdermott, J. (1982). Domain knowledge and the design process. Design studies, 3(1), 31$36 . \quad$ https://doi.org/10.1016/0142694X(82)90077-1

Nisbett, R. E. \& Norenzayan, A. (2002). Culture and cognition. In D. L. Medin (Ed.), Stevens' Handbook of Experimental Psychology, Third Edition, New York: John Wiley \& Sons. https://deepblue.lib.umich.edu/bitstream/h andle/2027.42/91934/culture_and_cognitio n.pdf\%3Bsequence $=1$

Oxman, R. E. \& Oxman, R. M. (1992). Refinement and adaptation in design cognition. Design studies, 13(2), 117-134. https://doi.org/10.1016/0142-694X(92)90259D

Oxman, R. E. (1990a). Design shells: A formalism for prototype refinement in knowledgebased design systems. Artificial intelligence in engineering, 5(1), 2-8. https://doi.org/10.1016/0954-1810(90)900308

Oxman, R. E. (1990b). Prior knowledge in design: a dynamic knowledge-based 
model of design and creativity. Design studies, 2(1), 17-28. https://doi.org/10.1016/0142-694X(90)90011Z

Oxman, R. E. (1992). Refinement and adaptation in design cognition. Design Studies. Vol. 13. No. 2.

Oxman, R. E. (1994). Precedents in design: a computational model for the organization of precedent knowledge. Design studies, 15(2), 141-157. https://doi.org/10.1016/0142694X(94)90021-3

Oxman, R. E. (1996). Design by rerepresentation: a model of visual reasoning in design. Design studies, 18(4), 329-347. https://www.academia.edu/5837938/Oxm an_Rivka_1997_Design_by rerepresentation a model of visual reasonin g_in_design

Oxman, R. E. (1999). Educating the designerly thinker. Design studies, 20(2), 105-122. https://doi.org/10.1016/S0142694X(98)00029-5

Oxman, R. E. (2001). The mind in design: a conceptual framework for cognition in design education. In C. Eastman, W. Newstetter \& M. McCracken (Eds.), Design knowing and learning: Cognition in design education, (pp. 269-295). Oxford: Elsevier Books.

https://www.elsevier.com/books/designknowing-and-learning-cognition-in-designeducation/eastman/978-0-08-043868-9

Oxman, R. E. (2004). Think-maps: teaching design thinking in design education. Design studies, 25(1). 63-91. https://doi.org/10.1016/s0142694X(03)00033-4

Oyserman, D., Sorensen, N., Reber, R. \& Chen, S. X. (2009). Connecting and separating mind-sets: culture as situated cognition. Journal of personality and social psychology, 97(2), 217-235. http://dx.doi.org/10.1037/a0015850

Petruccioli, A. (1998). Typological process and design theory. MA: Aga Khan Program for Islamic Architecture. https://archnet.org/publications/4240

Purcell, A. T. \& Gero, J. (1991). The effects of examples on the results of design activity. In J. S. Gero (Ed.), Artificial intelligence in design aid. Oxford, UK: ButterworthHeinemann. https://doi.org/10.1016/09507051(92)90026-C

Shore, B. (1996). Culture in mind: cognition, culture and the problem of meaning. Oxford: Oxford University Press. https://global.oup.com/academic/produc t/culture-in-mind9780195095975? $\mathrm{cc}=\operatorname{tr} \&$ lang=en\&

Simon, H. A. (1973). The structure of ill-structured problems. Artificial intelligence, 4, 181-201. https://doi.org/10.1016/0004-3702/73)90011$\underline{8}$

Suwa, M., Purcell, T. \& Gero, J. (1998). Macroscopic analysis of design processes based on a scheme for coding designers' cognitive actions. Design studies, 19(4), 45583. 694X(98)00016-7 https://doi.org/10.1016/S0142-

Uysal Ürey, Zeynep Çiğdem. (2019). The Sociocultural Role of Architectural Types: Cultural Sustainability in Architecture and the Possibility of Convention. GRID Architecture, Planning and Design Journal, $2 \quad$ (1), 83-99. http://dergipark.org.tr/grid/issue/42546/491 $\underline{782}$

Uysal, Zeynep Çiğdem. (2012). Architectural Type as a Cultural Schema and Its Cognitive Use in Architectural Design: An Analysis of the Aga Khan Award Winning Dwellings in Turkey (1970-2008), Unpublished PhD Dissertation, North Carolina State University, Raleigh. https://repository.lib.ncsu.edu/bitstream/ha ndle/1840.16/7522/etd.pdf? sequence $=2$

Wertsch, J. (Ed.). (1985). Culture, communication and cognition: Vyogotskian perspectives. Cambridge: Cambridge University Press. https://trove.nla.gov.au/work/6991064?a\&v ersionld $=45241165$ 